\title{
Simulation and HRI Recent Perspectives with the MORSE Simulator
}

\author{
Séverin Lemaignan ${ }^{1}$, Marc Hanheide ${ }^{2}$, Michael Karg ${ }^{3}$, Harmish Khambhaita ${ }^{4}$, \\ Lars Kunze $^{5}$, Florian Lier ${ }^{6}$, Ingo Lütkebohle ${ }^{7}$, and Grégoire Milliez ${ }^{4}$ \\ 1 CHILI Lab, EPFL, Lausanne, Switzerland \\ 2 Centre for Autonomous Systems, University of Lincoln, United Kingdom \\ 3 IAS, Technische Universität München, Germany \\ 4 LAAS/CNRS, Université de Toulouse, France \\ 5 Intelligent Robotics Lab, University of Birmingham, United Kingdom \\ ${ }^{6}$ CITEC, Bielefeld University, Germany \\ 7 Machine Learning and Robotics Lab, Universität Stuttgart, Germany
}

\begin{abstract}
Simulation in robotics is often a love-hate relationship: while simulators do save us a lot of time and effort compared to regular deployment of complex software architectures on complex hardware, simulators are also known to evade many of the real issues that robots need to manage when they enter the real world. Because humans are the paragon of dynamic, unpredictable, complex, real world entities, simulation of human-robot interactions may look condemn to fail, or, in the best case, to be mostly useless. This collective article reports on five independent applications of the MORSE simulator in the field of human-robot interaction: It appears that simulation is already useful, if not essential, to successfully carry out research in the field of HRI, and sometimes in scenarios we do not anticipate.
\end{abstract}

\section{Introduction}

The use of simulators for human-robot interaction (HRI) encompasses a variety of use-cases, from prototyping through evaluation to anticipatory simulation at runtime. It however suffers from a specific integration problem: Simulation in HRI requires to model robots in all their complexity plus a mean of representing and interacting with human agents. We therefore believe that an important stepping stone for a wider use of simulation in HRI is the availability of an integrated, easy-to-use framework that can encompass all currently important use-cases, and that provides an integration interface for developers and end-users of HRI simulation. In particular, we feel that it must be both easy to install and use, and offer adequate domain abstractions to facilitate development and integration. This paper presents how recent work using the Modular OpenRobots Simulation Engine [2] (MORSE, figure 1) attempts to address this challenge.

We will first review the range of current use-cases for simulation in HRI, then introduce MORSE with a focus on its HRI specific features, and finally demonstrate and discuss MORSE's versatility through several case studies. The 


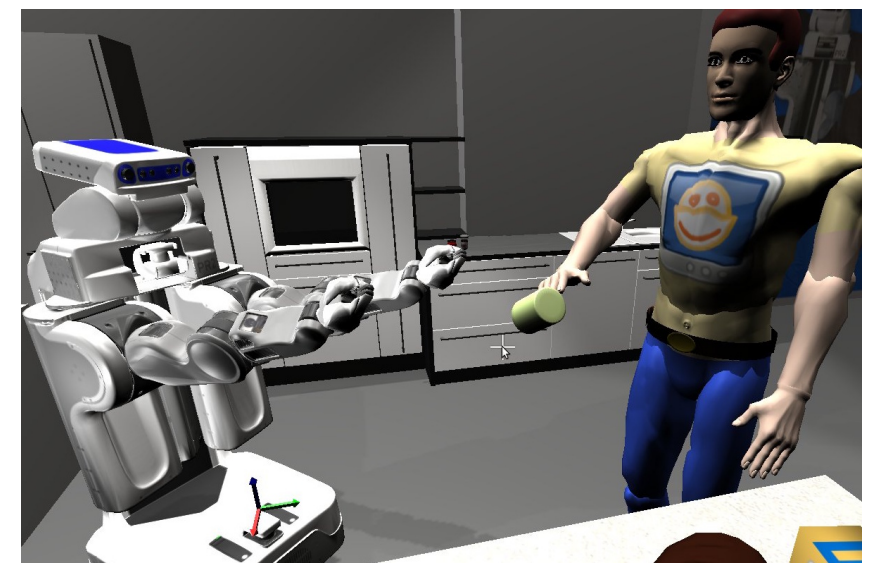

Fig. 1. Simulation and HRI: A PR2 and a human avatar in MORSE.

case studies also illustrate the collective nature of this article: We report on contributions and experiences in human-robot interaction simulation from five unrelated projects, conducted by different people in different organizations, only sharing the MORSE simulator as common simulation platform.

\section{HRI and simulation}

Applications of simulation in HRI In the HRI literature, several distinct goals for the use of simulation can be discerned. Without claim to completeness, we categorize them into 1) prototyping, 2) human modeling, 3) interactive simulation, and 4) anticipatory simulation.

The most well-known use-case is probably prototyping: The use of a simulator to reconstruct and run experiments in a simulated target situation prior to realworld evaluation. Apart from convenience, reasons to do so include simulation of unsafe situations (e.g. navigation in narrow spaces $[18,9]$ or crowds [6]), and exploration of edge cases (e.g. humans not paying attention $[10,5]$ ).

Human modeling is one way of realizing human agents in simulation. [3] present for example a pedestrian model which has been evaluated against a large-scale database of recorded human movements. When detailed motion or other actions (such as speaking) is required, cognitive models have often been used [19], and recently also in HRI [22]. It is probably safe to say that such models are still far from general, but already quite useful for specific situations.

A problem with these approaches is the significant up-front effort required for modeling. Therefore, some research has explored the use of game engines in what we call interactive simulation: a real human controls a simulated human avatar interactively. While not fully automated, it allows reliable capture of interaction data for later analysis. This has been used for a long time in tele-operation settings [24] and also more recently for so-called "crowd-sourcing" work [1]. 
A very different use of simulation is anticipatory simulation. Here, a simulator is used at runtime, to be able to quickly explore the likely consequences of robotic actions. This uses simulation engines mainly to support spatial computations, e.g. to compute social metrics such as walking comfort [9] or proxemics [7]. The general goal is to enable the system to choose an action based on these metrics.

We believe it is clear that these use-cases benefit from each other. Particularly prototyping requires models, which could be manually specified, learned from real-world data, or learned interactively through the simulator.

Simulators for HRI Softwares used for HRI simulation are currently fairly diverse, and can be distinguished by their use-cases. Prototyping work often uses "standard" robot simulators such as USARSim [14] (commonly used for rescue robotics applications but also beyond), or Gazebo [11] (though Gazebo's human agent support is currently limited) and MORSE [2].

In contrast, work in models or more advanced use-cases such as interactive or anticipatory simulation currently uses custom software - this is true for all of the papers cited in the previous sections at least. The pedestrian modeling community seems to share some tools, e.g. the work by Treuille et al [23] is known to have been re-used, but it has no connection to robot simulators.

Both standard robot simulators and pedestrian simulators use fairly coarse human models. In contrast, work in the Embodied Virtual Agent (EVA) community usually provides higher-level functionality, such as simulated emotion dynamics, behavior generation based on action primitives, conversational dialogue systems, and up to cognitive simulations. However, integrating these into a coherent system with an acceptable interface remains challenging [4].

As stated before, we think that the integration of these diverse functionalities into standard robot simulators would be an ideal next step, making specialized tools available to a much wider audience, and thus likely also identifying new avenues for improvements.

\section{HRI and the MORSE simulator}

All five projects that are presented in this article rely on the domain-independent MORSE simulator as simulation platform. MORSE is an open-source tool developed for academic robotic research with contributions from over 15 institutions worldwide. It extends the Blender Game Engine, a 3D engine which features shader-based 3D rendering and physics simulation (BULLET physics engine). This allows for semi-realistic simulation of complex environments. The MORSE components (sensors and actuators) exchange data with the robotics software via middleware bindings (Software In The Loop architecture). Four middlewares designed for robotics are currently supported, including ROS and YARP, as well as a generic socket-based protocol. This design aims at providing a seamless experience when switching back and forth between the simulator and the physical robot. Standard robotic platforms, actuators and sensors (more than 50 components) are provided and enable fast creation of simulation scenarios, while custom components and behaviors can be added via simple Python scripts. 
MORSE also introduces a concept of abstraction levels: sensors and actuators may expose several levels of abstraction, corresponding to different level of realism. For instance, users may choose if the odometry sensor returns only a curvilinear distance, a $d X, d Y, d Z$ differential vector, or the absolute position of the robot (integrated odometry). This allows users that are testing low-level components to do so, while users working at higher abstraction levels (typically in HRI) do not have to run full robotic software stacks (and thus, benefit from a lighter environment) and can work in a more deterministic environment. This feature can be finely controlled, on a per-component basis.

For HRI applications, MORSE provides a human avatar that can be fully controlled (displacement, gaze, grasping of objects, interactions with the environment like turning lights on, opening drawers, doors...) from a first-person-shooter perspective. This enables the researcher to quickly setup and test human-robot interactions with a tele-operated human model, hence with realistic human behaviors. As presented in [13], the human avatar can be controlled using a Kinectlike device. The same avatar can also be programmatically controlled by external scripts, like any robot in MORSE. With standard MORSE actuators like the waypoint actuator, the researcher can for instance pre-define paths that the human avatar will follow in a simulated environment.

\section{HRI Simulation : Five Scenarios}

To illustrate how simulation can support research in HRI, we present in the next sections five case-studies. The first three scenarios, Situation Assessment for HRI and Simulated Feedback, An Expectations Framework for Domestic Robot Assistants and Preliminary Testing of Human-Aware Navigation Planner illustrate the typically use-case for simulation: rather complex virtual environments are created where human presence plays a central role, and HRI algorithms are tested in a convenient and repeatable way. Note that, while we introduce here simulation-only scenarios, they all are test-cases of experiments that have been conducted on real robots: simulation is used here to support real-world deployments.

The fourth scenario, Data Acquisition through Automatic Scene Generation shows how simulation is used as an alternative source of input to train robots to behave in human environments, and the last scenario, Automated Execution of Prototype HRI Experiments, presents how the simulator can be used to provide automatic testing of human-aware behaviors, fully integrated to the software development workflow. Each of the presentations follow the same structure: we first introduce the scenario, we then highlight how simulation has been leveraged and its benefits, and we finally mention some of the shortcomings of the tool.

\subsection{Situation Assessment for HRI and Simulated Feedback}

When studying human-robot interaction, understanding the environment in which agents will interact is a key issue. In this first application, MORSE provides 


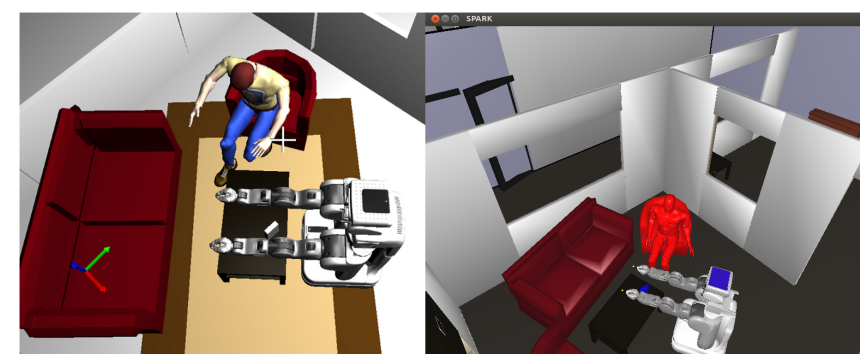

Fig. 2. On the left side, the MORSE environment ; on the right side, the same environment, as perceived by the robot in the SPARK situation assessment module.

a virtual environment that we use to harness situation assessment algorithms (performed by a software called SPARK [17], for SPAtial Reasoning and Knowledge) that also include human-centered perspective taking. The robot updates its knowledge in SPARK using its own position, human position and objects seen through abstracted, symbolic cameras provided by MORSE (so-called semantic cameras). In this particular scenario (figure 2), the human is sitting in a couch and ask the robot to bring specific objects that may be in another room (Pick-Place-Carry task).

Benefits of the simulation The direct benefits of relying on MORSE for the development of the situation assessment algorithms is the low-cost of deployment (manual testing on physical systems is labour-intensive), as well as the repeatability of the experimental conditions, important to assess the algorithmic improvements. Also, relying on MORSE effectively supports collaboration between the partners involved in this project (MaRDi project $\left.{ }^{8}\right)$ : our partners are also using MORSE simulation to test their software and collect data with the same environment in their laboratory, where they focus on dialog processing. They can train their dialog system using MORSE feedback to test the robot behaviors [16].

\subsection{An Expectations Framework for Domestic Robot Assistants}

In this scenario, an apartment is simulated in which a domestic service robot is living together with a person (figure 3). A PR2 robot is controlled via ROS and the CRAM reactive plan language, which is used on several other real robots. The robots' duty is to observe the person performing different activities and detect unexpected situations based on the validation of different types of expectations [8]. The detection of such unexpected behavior can help future domestic service robots to better assess situations and adapt their actions to human behavior.

Benefits of the simulation The use of the MORSE simulator enabled us to set up a large testbed by reusing the real robot control layer via the ROS mid-

\footnotetext{
${ }^{8}$ http://mardi.metz.supelec.fr
} 


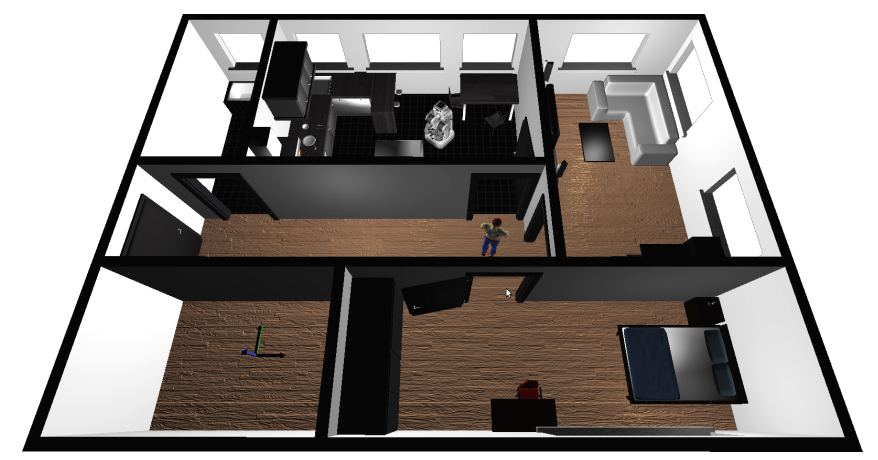

Fig. 3. A simulated apartment with a domestic service robot and a person.

dleware and easy-to-generate unpredictable human behaviors using the human component of MORSE. Setting up of such an apartment in a real-world setting, together with a suitable sensor setup and a reliable robot control, would not only introduce huge costs but would also be a time-consuming task which can distract researchers from their actual research focus. The use of the simulated scenario enables us to gain many insights into the problem domain in a scenario that would not been possible within our project, while the algorithms were eventually validated on the real robot, inside a smaller real-world environment.

The human component of MORSE enabled us to test and validate our approach dynamically in a variety of situations. Since it can be controlled in realtime like in a 3D computer game while at the same time, a robot can be simulated by state-of-the-art components, it is possible to generate a multitude of situations on which the robot has to react. This greatly supported our project to gain insights about our approach, detect weak points and make improvements.

\subsection{Preliminary Testing of Human-Aware Navigation Planner}

To evaluate the improvements in the human-aware navigation planner developed at LAAS we carried out a user study. An experiment was set up, where a robot encounters a human crossing its path (at $90^{\circ}$ angle to each other) while the robot is moving forward to its navigation goal. For preliminary testing of the planning algorithm, our lab area was simulated in MORSE. This simulated environment was extensively used to review the algorithm before it was deployed on the PR2 robot to carry out real-world experiments (whose results have been published in [21]).

Benefits of the simulation Development of human-robot interaction algorithms often require iterative process of prototyping, testing and reviewing. Setting up and experiment and testing of robot navigation algorithms especially for large environments involving humans is time consuming and is subject to availability of lab resources while working in a shared lab between different groups of researchers. Full support of the PR2 robot model among others, availability of a 
human model, and a convenient way of setting up experiment environment using Blender software were the most prominent features for choosing MORSE as the simulation environment for these experiments. Since MORSE already provides ROS bindings for the PR2 robot and human pose it requires minimal effort to switch between real-world and simulated environments.

As a consortium member in the EU project SPENCER ${ }^{9}$, we plan to develop novel algorithms for robot navigation in large populated environments, e.g. airports. In the future we plan to use MORSE to simulate such large environment with multiple human models. This will certainly push the limits of simulation for HRI and hopefully provide new benchmarks.

\subsection{Data Acquisition through Automatic Scene Generation}

This fourth study proposes a different perspective on the role of simulation in HRI: simulating credible human environments to train systems to appropriately react to them: autonomous mobile robots that are to help and assist people in care homes, households, and at other workplaces have to understand how human activities affect the dynamics of objects in the environment. That is, robots need to know, when, where and how people manipulate objects and how they arrange and structure them in space. In the context of the STRANDS project ${ }^{10}$ we aim for robots that understand the long-term, spatio-temporal relationships of objects and activities of people. In the scenario described here, we looked in particular at learning qualitative spatial relations of objects on office desks. As an accurate classification and pose estimation of objects on real-world office desks is still a challenging and difficult task for current robot perception systems we acquired a data set of object arrangements using the MORSE simulator. For this, we first bootstrapped an object statistics from manually labeled images of real office desks, and secondly, automatically generated a set of physically possible desktop scenes (figure 4). Based on the generated data we learned relational models of object arrangements on desks. The learnt models enabled a robot to predict the position of an object given a landmark. We employed these models to effectively guide a simulated and a real robot in object search tasks and evaluated its performance [12].

Benefits of the simulation First, the automatic scene generation (made easy by the use of Python to "program" the simulation scenes) and annotation of object arrangements in simulation is useful for the acquisition of large amounts of data over short periods of time. The generated data enabled us to design, implement and to evaluate our methods for predicting object locations before having a real-world data set in place. Secondly, the generation of object arrangements can increase the variability of scenes in human-robot experiments in general. Given the dynamics of objects in the real world it is important not to oversimplify human-robot experiments in simulated environments but make them as realistic as possible (in a controlled way). Finally, in future work, we plan

\footnotetext{
${ }^{9}$ http://www. spencer.eu

${ }^{10}$ http://www.strands-project.eu
} 

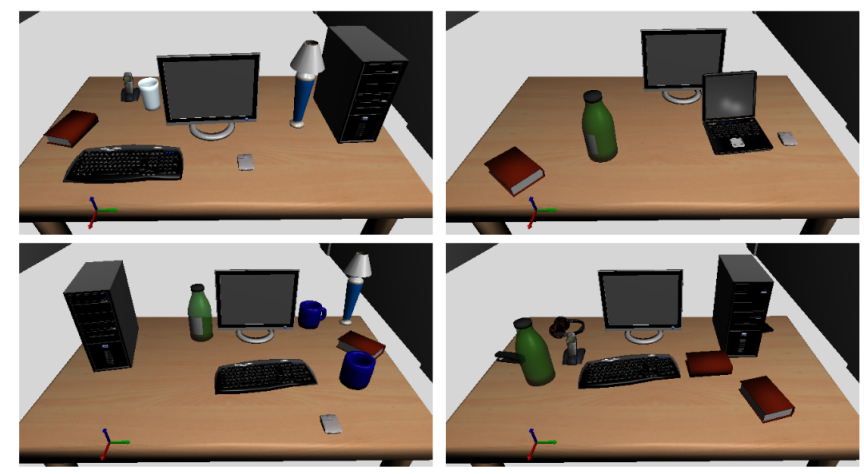

Fig. 4. Automatically generated scenes of office desks.

to use the generated desktop scenes in web-applications to crowdsource Natural Language descriptions of object arrangements and commands for robots from Internet users.

\subsection{Automated Execution of Prototype HRI Experiments}

In human-robot interaction studies, robots often indicate behavioral variability that may influence the experiment's final outcome. However, manual testing on physical systems is usually the only way to prevent this, but remains labour-intensive. To tackle this issue, we introduced early automated prototype testing [15] that consists of: a simulation environment, a software framework for automated bootstrapping of prototype systems, execution verification of system components, automated result assessment of experiments and a Continuous Integration (CI) server to centralize experiment execution. In our setup we bootstrap and execute a simulated prototype system on a CI server and assess the results in each run. In this particular scenario, a robot must report the location of a virtual human in a domestic environment. Both the robot and the human are moving in the scene and meet in front of a table.

The goal of this simulation setup is to incrementally decrease the level of abstraction until a satisfactory/sufficient degree of "realism" to make an assumption about real world behavior, is reached - in an integrated and continuous approach. In order to achieve this goal, we make use of two essential MORSE features: a) the human avatar that can be steered (set waypoints) interactively via middleware and $b$ ) a semantic camera that extracts the location of a specific entity in the simulation environment. The semantic camera is attached to the robot. If the human enters the robot's field of view, the location is reported and sent via middleware. After each CI run, the recorded movement trajectory of the human avatar is assessed (plotted) and archived. We have explicitly chosen to simplify the extraction of the location of the human to acquire a ground truth in the first iterations of the simulation. As an example, a system component (running outside of the simulation) that is intended to classify whether there is 
a human in front of a robot, by fusing multiple sensory inputs, can be evaluated based on this ground truth. Subsequently, we are able to exchange/add diverse virtual sensors, i.e. add a simulated laser scanner to build a person hypothesis for instance, thus gradually develop, assess and implement more complex scenarios.

Benefits of the simulation First of all, the interactive (remotely controllable) human avatar is useful to include a dynamic, yet not too realistic, human component in this setup. Secondly, the level of abstraction of different sensors, i.e. semantic camera versus virtual laser scanner enables us to gradually raise the level of complexity/realism and test different algorithms based on abstract and almost realistic sensor inputs. Lastly, the chance to deploy MORSE in a Continuous Integration environment, i.e. automatically run simulation scripts, generates an additional benefit.

\section{Discussion: Towards Unification}

While the five scenarios that we present here implement different use-cases, they actually cover similar approaches, while relying on the same simulator: study 2.1 shows how MORSE can be thought as a computation engine, 2.2 exploits the human agent in a computer game style, 2.3 uses MORSE for assessing and tuning the performance of algorithms, and scenario 2.4, while somewhat unique, still share similarities with Garrell et al., in that a model for object positions is trained on real-world data. Finally, the use-case presented in 2.5 proposes a different approach, with a focus on continuous testing, and can arguably be seen as the natural progression of using simulators for evaluation, extended here to cover HRI scenarios.

From this perspective, one may consider that the experiments recently conducted in the MORSE community around the simulation of HRI applications constitute the first steps towards building an unifying platform for HRI simulation, with two additional features: its programmability (simulation scenarios are Python scripts) and its concept of abstraction levels that provides an effective way to focus simulation on a particular problem by hiding irrelevant simulation artifacts.

These diverse use-cases support the idea that simulation is not only actually useful as a support tool for development of human-robot applications, but also enables new research techniques in HRI. Continuous Integration illustrates this point: while HRI experiments are considered as notoriously difficult to deploy, test and repeat, we show here how a simulator may enable automated testing of more and more complex scenarios, including long-term interaction.

Several issues are also raised and must be clearly stated. In its current state, the MORSE simulator provides only an incomplete model of the environment. Sounds/speech models are incomplete, and human models do not yet provide good enough accuracy, both at the level of the user interface (some actions can not be done with the interactive avatar), and at the simulation level (poor/missing walking cycles for instance). Finally, the overall convenience of MORSE for HRI could be improved, for instance by providing more assets (fur- 
nitures, objects) related to human environments. These issues, that are mostly technical and could be addressed at the software level, show that simulators dedicated to HRI application still need to mature. In that regard, the next section presents some of the directions that are currently researched.

\section{The next steps}

Several noteworthy developments related to HRI are currently shaping up in the MORSE community. We outline below some of them, that suggest new applications that we believe are relevant to HRI research.

A first line of investigation relates to the procedural generation of a variety of realistic human models. MAKEHUMAN is such an open-source tool that generates anatomically, kinetically and visually realistic human models. This software has a tight integration with Blender, and MORSE is soon to provide as well seamless integration with MAKEHUMAN models. This will bring a wide range of characters to feed the simulations, and extend testing environments with gender/size/age/skin color variances.

Besides being able to control a human avatar in simulation programmatically and deterministically, the possibility to automatically generate believable and realistic crowd behaviors is being explored. In this context, the objective is to adopt in MORSE technologies previously developed for computer games to generate trajectories that control the MORSE avatars. Based on the idea of social forces, the work of [20] is to be adapted to provide believable and realistic movement of several humans within MORSE. This implementation would provide a more realistic and dynamic environment to study human-robot spatial interaction and to provide a testbed for human-aware motion planning, to give two exemplary use-cases.

Another line of investigation looks at embedding the researcher into the robotic simulation. The purpose of such efforts is to provide a life-like immersive simulation environment that would allow at the same time ecologically valid human behaviors and repeatable, lightweight interaction settings. In [13], we presented how a human agent could interact with a virtual robot through a deictic interface based on a Kinect. Two distinct projects are currently looking into extending this approach, one (at Bielefeld University) aiming at integrating emerging Virtual Reality devices (like Occulus Rift) with MORSE, the other one (MarDI project) developing a virtual reality cave, that include $360^{\circ}$ projections and spatialized sound.

Also often suggested, the on-line deployment of HRI simulations could efficiently support large scale HRI studies. The simulator and specific interaction scenarios would be embedded in a dedicated webpage and users would control a human avatar from their webbrowsers. This would potentially enable collection of large behavioral datasets. While MORSE development in that direction has yet to start, Breazeal et al. presented an initial attempt in that direction in [1] and the Gazebo simulator features a limited WebGL client that act as a proof-of-concept of on-line robotic simulation. 


\section{Conclusion}

These examples and ideas hopefully give a picture of the lively landscape of the "Simulation for HRI" community, that has built itself around the MORSE simulator. In the introduction, we mentioned how simulation in HRI had to address in parallel constraints stemming from robotic simulation and virtual agent simulation, while remaining a lightweight, easy-to-use tool. We are certainly not yet there, much remains to be imagined, refined and achieved. Yet MORSE is already deployed in several institutions as a platform that efficiently supports research in human-robot interaction. As an open-source project, we strive for new use-cases and ideas, and warmly welcome researchers that would like to join the effort.

\section{Acknowledgment}

This research has received funding from the European Union (FP7/2007-2013) under grant agreements FP7-600623 (STRANDS) and FP7-600877 (SPENCER).

\section{References}

1. C. Breazeal, N. DePalma, J. Orkin, S. Chernova, and M. Jung. Crowdsourcing human-robot interaction: New methods and system evaluation in a public environment. Journal of Human-Robot Interaction, 2(1):82-111, 2013.

2. G. Echeverria, S. Lemaignan, A. Degroote, S. Lacroix, M. Karg, P. Koch, C. Lesire, and S. Stinckwich. Simulating complex robotic scenarios with MORSE. In Simulation, Modeling, and Programming for Autonomous Robots (SIMPAR), 3rd International Conference on, pages 197-208, 2012.

3. A. Garrell and A. Sanfeliu. Model validation: Robot behavior in people guidance mission using DTM model and estimation of human motion behavior. In IEEE Transactions on Intelligent Robots and Systems (IROS), pages 5836-5841. IEEE, 2010.

4. J. Gratch, J. Rickel, E. André, J. Cassell, E. Petajan, and N. Badler. Creating interactive virtual humans: Some assembly required. Intelligent Systems, IEEE, $17(4): 54-63,2002$.

5. J. Guzzi, A. Giusti, L. M. Gambardella, G. Theraulaz, and G. A. Di Caro. Humanfriendly robot navigation in dynamic environments. In IEEE International Conference on Robotics and Automation (ICRA), pages 423-430. IEEE, 2013.

6. P. Henry, C. Vollmer, B. Ferris, and D. Fox. Learning to navigate through crowded environments. In IEEE International Conference on Robotics and Automation (ICRA), pages 981-986. IEEE, 2010.

7. G. Hoffman and C. Breazeal. Effects of anticipatory perceptual simulation on practiced human-robot tasks. Autonomous Robots, 28(4):403-423, 2010.

8. M. Karg and A. Kirsch. An expectations framework for domestic robot assistants. In Conference on Advances in Cognitive Systems, pages 77-92, 2013.

9. H. Kidokoro, T. Kanda, D. Brscic, and M. Shiomi. Will I bother here? - A robot anticipating its influence on pedestrian walking comfort. In 8th ACM/IEEE International Conference on Human-Robot Interaction (HRI), pages 259-266. IEEE, 2013. 
10. R. A. Knepper and D. Rus. Pedestrian-inspired sampling-based multi-robot collision avoidance. In 21st IEEE International Symposium on Robot and Human Interactive Communication (RO-MAN), pages 94-100. IEEE, 2012.

11. N. Koenig and A. Howard. Design and use paradigms for Gazebo, an opensource multi-robot simulator. In IEEE/RSJ International Conference on Intelligent Robots and Systems (IROS), volume 3, pages 2149-2154. IEEE.

12. L. Kunze, K. Kumar, and N. Hawes. Indirect object search based on qualitative spatial relations. In IEEE International Conference on Robotics and Automation (ICRA), May 31 - June 72014.

13. S. Lemaignan, E. G., M. Karg, M. Mainprice, A. Kirsch, and R. Alami. Humanrobot interaction in the MORSE simulator. In 7th ACM/IEEE International Conference on Human-Robot Interaction (HRI), 2012. Late Breaking Reports.

14. M. Lewis, J. Wang, and S. Hughes. USARSim: Simulation for the study of humanrobot interaction. Journal of Cognitive Engineering and Decision Making, 1(1):98120, 2007.

15. F. Lier, I. Lütkebohle, and S. Wachsmuth. Towards automated execution and evaluation of simulated prototype HRI experiments. In 9th ACM/IEEE Conference on Human-Robot Interaction (HRI), 2014. Late Breaking Reports.

16. G. Milliez, E. Ferreira, M. Fiore, R. Alami, and P. Lefèvre. Simulating human-robot interactions for dialogue strategy learning. In IEEE/RSJ International Conference on Intelligent Robots and Systems, (SIMPAR2014), 2014.

17. G. Milliez, M. Warnier, A. Clodic, and R. Alami. A framework for endowing interactive robot with reasoning capabilities about perspective-taking and belief management. In Proceedings of the 23rd IEEE International Symposium on Robot and Human Interactive Communication, 2014.

18. E. A. Sisbot, L. F. Marin-Urias, R. Alami, and T. Simeon. A human aware mobile robot motion planner. Transactions on Robotics, 23(5):874-883, 2007.

19. R. Sun. Cognition and multi-agent interaction: From cognitive modeling to social simulation. Cambridge University Press, 2006.

20. O. Szymanezyk, T. Duckett, and P. Dickinson. Agent-based crowd simulation in airports using games technology. In Transactions on Computational Collective Intelligence VIII, pages 192-213. Springer Berlin Heidelberg, 2012.

21. Thibault Kruse, Harmish Khambhaita, Alexandra Kirsch, and Rachid Alami. Evaluating directional cost models in navigation. In 9th ACM/IEEE Conference on Human-Robot Interaction (HRI), Bielefeld, Germany, 2014.

22. G. Trafton, L. Hiatt, A. Harrison, F. Tamborello, S. Khemlani, and A. Schultz. ActR/E: An embodied cognitive architecture for human-robot interaction. Journal of Human-Robot Interaction, 2(1):30-55, 2013.

23. A. Treuille, S. Cooper, and Z. Popović. Continuum crowds. In ACM Transactions on Graphics (TOG), volume 25, pages 1160-1168. ACM, 2006.

24. J. Wang, M. Lewis, S. Hughes, M. Koes, and S. Carpin. Validating USARsim for use in HRI research. Human Factors and Ergonomics Society Annual Meeting, 49(3):457-461, Sept. 2005. 